\title{
Magnetic Properties of Nano-Crystalline Barium Ferrite Synthesized by Different Synthesis Route
}

\author{
H. Ovalioglu ${ }^{a}$, H. Sozeri ${ }^{b}$, M. KABAer ${ }^{a}$ And I. KuCuK ${ }^{a, *}$ \\ ${ }^{a}$ Physics Department, Faculty of Arts and Sciences, Uludag University, Gorukle Campus 16059, Bursa, Turkey \\ ${ }^{b}$ TUBITAK-UME, National Metrology Institute, P.O. Box 54, TR-41470, Gebze, Kocaeli, Turkey
}

We have synthesized $\mathrm{Ba}_{1-x} \mathrm{La}_{x} \mathrm{Fe}_{12} \mathrm{O}_{19}(0 \leq x \leq 0.4)$ and $\mathrm{BaFe}_{12-y} \mathrm{Nd}_{y} \mathrm{O}_{19}(0 \leq y \leq 4)$ samples in ammonium nitrate melt and nitric acid. The effects of La and Nd doping on the microstructure and magnetic properties of $\mathrm{BaFe}_{12} \mathrm{O}_{19}$ were investigated. The highest coercive field was obtained for the sample having La content, $x=0.3$.

PACS numbers: 75.50.Gg, 75.50.Ss, 75.50.Ww, 61.72.U-

\section{Introduction}

The barium ferrite has attracted great interest in the magnetic material field and has been widely used as a permanent magnet because of its fairly large magnetocrystalline anisotropy and high Curie temperature, together with relatively large saturation magnetization, excellent chemical stability and corrosion resistivity. Various chemical routes were used to synthesize nanoparticles of Ba-hexaferrite: microemulsion [1], chemical co-precipitation [2], glass crystallization [3], combustion, sol-gel synthesis [4], hydrothermal synthesis [5], oxidation in nitric acid method [6], and ammonium nitrate melt (ANM) technique [7]. The magnetic properties of $\mathrm{BaFe}_{12} \mathrm{O}_{19}$ can be improved by the substitution of $\mathrm{Ba}^{2+}$ and/or $\mathrm{Fe}^{3+}$. In order to achieve this purpose, element substitution was widely used for the synthesis of magnetic materials with appropriate saturation magnetization and coercivity [8-13].

In this work, we aimed to synthesize single domain La and Nd doped barium ferrite nanoparticles with high saturation magnetization $\left(M_{\mathrm{s}}\right)$ and high coercivity $\left(H_{\mathrm{c}}\right)$ by the ANM and oxidation in nitric acid method.

\section{Experimental}

Appropriate amounts of $\mathrm{BaCO}_{3}, \mathrm{Fe}_{2} \mathrm{O}_{3}, \mathrm{Nd}_{2} \mathrm{O}_{3}$ and $\mathrm{La}_{2} \mathrm{O}_{3}$ powders of high purity $(99 \%)$ were weighed and mixed at different La and Nd. The mixed powder was put into the melted ammonium nitrate or nitric acid. The solution was mixed on a hot plate with magnetic stirrer until the liquid disappeared. Before grinding in an agate mortar for $15 \mathrm{~min}$, small amount of isopropyl alcohol was added to make wet grinding. The precursor was calcinated at $450{ }^{\circ} \mathrm{C}$ for $5 \mathrm{~h}$ to remove possible organic compounds. Then, the precursor was again grinded for

* corresponding author; e-mail: ikucuk@uludag.edu.tr
15 min with isopropyl alcohol and dried, pelletized under the pressure of $200 \mathrm{MPa}$. The pressed disc-shaped pellets (diameter $16 \mathrm{~mm}$, thickness $3 \mathrm{~mm}$ ) were sintered at $1000^{\circ} \mathrm{C}$ for $2 \mathrm{~h}$ in the air and then cooled in the furnace.

Structural analysis was done by scanning electron microscope (JEOL 6335F, Field Emission Gun). The magnetic properties of samples were examined at room temperature using vibrating sample magnetometer (LDJ Electronics Inc., Model 9600) with the maximum field up to $1.6 \mathrm{~T}$.

\section{Results and discussion}

Figure 1a,b shows the scanning electron microscopy (SEM) micrographs of the pure and La-doped samples calcinated at $1000{ }^{\circ} \mathrm{C}$ for $2 \mathrm{~h}$. It is clearly seen that the samples, Fig. 1a and b, have grains between $100 \mathrm{~nm}$ and $250 \mathrm{~nm}$, in the single domain limit $(<1 \mu \mathrm{m})$. Besides, the grains are distributed homogeneously without massive agglomerations.

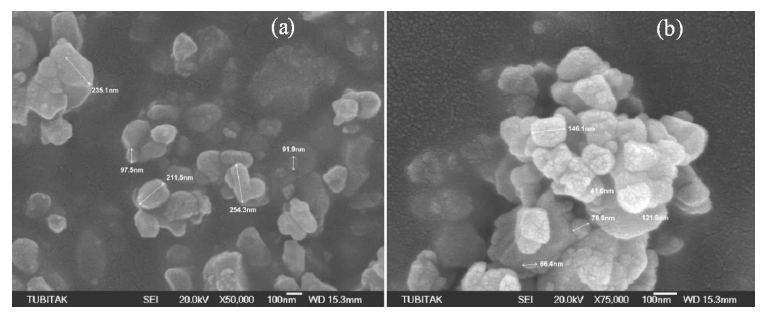

Fig. 1. SEM micrographs of the samples: (a) pure, (b) La amount of $x=0.3$.

The hysteresis loops of $\mathrm{Nd}$-substituted barium hexaferrites sintered at $1000^{\circ} \mathrm{C}$ show a behavior of the hard magnetic materials with high coercive field, as shown in Fig. 2. As seen from the figure, the $M_{\mathrm{s}}$ values decrease upon increasing $\mathrm{Nd}$ content, whereas the $H_{\mathrm{c}}$ values remain almost the same with $\mathrm{Nd}$ doping for 


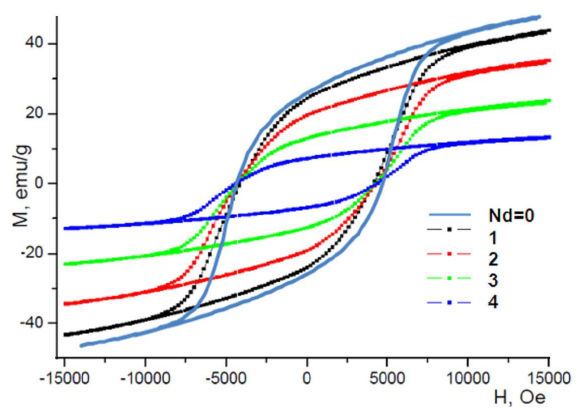

Fig. 2. The hysteresis loops of $\mathrm{BaFe}_{12-y} \mathrm{Nd}_{y} \mathrm{O}_{19}$ $(0 \leq y \leq 4)$ samples.

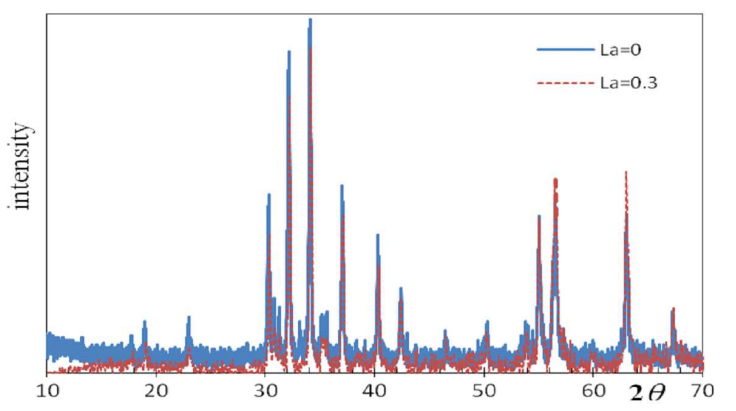

Fig. 3. XRD patterns of the $\mathrm{La}=0.0$ and $\mathrm{La}=0.3$ samples.

$\mathrm{BaFe}_{12-y} \mathrm{Nd}_{y} \mathrm{O}_{19}(1 \leq y \leq 4)$. The $\mathrm{X}$-ray diffraction patterns of $\mathrm{Ba}_{1-x} \mathrm{La}_{x} \mathrm{Fe}_{12} \mathrm{O}_{19}(x=0.0$ and $x=0.3)$ hexaferrite powders are shown in Fig. 3. The X-ray diffraction patterns show the samples to have a single $\mathrm{BaFe}_{12} \mathrm{O}_{19}$ phase. This indicates that $\mathrm{La}^{3+}$ ions were completely solved into the hexagonal crystal lattice. The variations of $M-H$ loops of the $\mathrm{Ba}_{1-x} \mathrm{La}_{x} \mathrm{Fe}_{12} \mathrm{O}_{19}(0 \leq x \leq 0.4)$ samples are also shown in Fig. 4. The $M_{\mathrm{s}}$ decreases and there is increase in coercivity with La amount in samples. The $H_{\mathrm{c}}$ becomes maximum for doping levels $x=0.3$ and 0.4 . However, $M_{\mathrm{s}}$ corresponding to $x=0.3$ is slightly higher than that of the latter one. Therefore, the optimum doping level seems to be $x=0.3$ to have the highest coercivity and high magnetization.

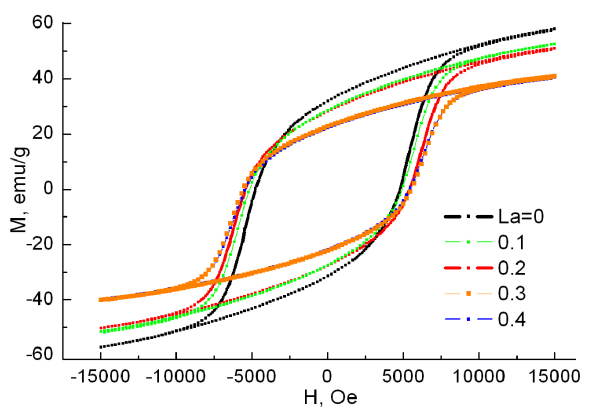

Fig. 4. The $M-H$ loops of $\mathrm{Ba}_{1-x} \mathrm{La}_{x} \mathrm{Fe}_{12} \mathrm{O}_{19}$ $(0 \leq x \leq 0.4)$ samples.

\section{Conclusion}

$\mathrm{Ba}_{1-x} \mathrm{La}_{x} \mathrm{Fe}_{12} \mathrm{O}_{19} \quad(0.0, \quad 0.1, \quad 0.2, \quad 0.3, \quad 0.4)$ and $\mathrm{BaFe}_{12-y} \mathrm{Nd}_{y} \mathrm{O}_{19}(1,2,3,4)$ ferrite powders were prepared and investigated. The microstructure and magnetic properties have been characterized. Comparing the La-doped barium ferrite with the undoped barium ferrite, the coercivity of the La-doped barium ferrite is higher than that of the undoped barium ferrite. However, the coercivity of $\mathrm{Nd}$-doped powders was remained nearly same. When the La amount is $x=0.3$, the coercivity of La-doped barium ferrite shows the maximum value. The saturation magnetization of the samples was found to decrease with increase in the La and Nd content. The results showed that $\mathrm{La}$ and $\mathrm{Nd}$ doping to $\mathrm{BaFe}_{12} \mathrm{O}_{19}$ changes the magnetic properties of this material significantly.

\section{Acknowledgments}

This work was supported by the commission of Scientific Research projects of Uludag University, project number $\operatorname{UAP}(\mathrm{F})-2010 / 19$.

\section{References}

[1] X. Lui, J. Wang, L.-M. Gan, S.-C. Ng, J. Ding, J. Magn. Magn. Mater. 184, 344 (1998).

[2] S.E. Jacobo, C. Domingo-Pascal, R. Rodriguez-Clemente, M.A. Blesa, J. Mater. Sci. 32, 1025 (1997).

[3] R. Muller, R. Heirgeist, H. Steinmetz, N. Ayoub, M. Fujisaki, W. Schuppel, J. Magn. Magn. Mater. 201, 34 (1999).

[4] J. Huang, H. Zhuang, W.L. Li, Mater. Res. Bull. 38, 149 (2003).

[5] X. Liu, J. Wang, L.M. Gan, S.C. Ng, J. Magn. Magn. Mater. 195, 452 (1999).

[6] H. Sozeri, J. Magn. Magn. Mater. 321, 2717 (2009).

[7] H. Sozeri, N. Ghazanfari, Mater. Chem. Phys. 113, 977 (2009).

[8] Z.H. Hua, S.Z. Li, Z.D. Han, D.H. Wang, M. Lu, W. Zhong, B.X. Gu, Y.W. Du, Mater. Sci. Eng. A-Struct. 448, 326 (2007).

[9] N.H. Hur, J.Y. Park, J. Dho, S.J. Kim, E.K. Lee, IEEE Trans. Magn. 40, 2790 (2004).

[10] R. Grössinger, C. TellezBlanco, M. Küpferling, M. Müller, G. Wiesinger, Physica B 327, 202 (2003).

[11] P. Tenaud, A. Morel, F. Kools, J.M. Le Breton, L. Lechevallier, J. Alloy Comp. 370, 331 (2004).

[12] R. Grössinger, M. Küpferling, J.C. Tellez Blanco, G. Wiesinger, M. Müller, G. Hilscher, M.W. Pieper, J.F. Wang, I.R. Harris, EIEEE Trans. Magn. 39, 2911 (2003).

[13] D. Lisjak, M. Drofenik, J. Eur. Ceram. Soc. 24, 1841 (2004). 